\title{
Nonlinear Schrödinger-Helmholtz equation as numerical regularization of the nonlinear Schrödinger equation
}

\author{
Yanping Cao ${ }^{1}$, Ziad H Musslimani ${ }^{2}$ and Edriss S Titi ${ }^{3,4}$ \\ ${ }^{1}$ Department of Mathematics, University of California, Irvine, CA 92697-3875, USA \\ 2 Department of Mathematics, Florida State University, Tallahassee, FL 32306, USA \\ ${ }^{3}$ Department of Mathematics and Department of Mechanical and Aerospace Engineering, \\ University of California, Irvine, CA 92697-3875, USA \\ E-mail: ycao@math.uci.edu,musliman@mail.math.fsu.edu, etiti@math.uci.edu and \\ edriss.titi@weizmann.ac.il
}

Received 27 June 2007, in final form 7 February 2008

Published 26 March 2008

Online at stacks.iop.org/Non/21/879

Recommended by F Merle

\begin{abstract}
A regularized $\alpha$-system of the nonlinear Schrödinger (NLS) equation with $2 \sigma$ nonlinear power in dimension $N$ is studied. We prove short time existence and uniqueness of solution in the case $1 \leqslant \sigma<\frac{4}{N-2}$. And in the case $1 \leqslant \sigma<3$ (when $N=1$ ) or in the case $1 \leqslant \sigma<\frac{4}{N}$ (when $N>1$ ) we show global in time existence of solutions. When $\alpha \rightarrow 0^{+}$, the solutions of this regularized system will converge to the solutions of the classical NLS in the appropriate range when the latter exists. Consequently, we propose this regularized system as a numerical regularization to shed light on the profile of the blow-up solutions of the original NLS equation in the range $\frac{2}{N} \leqslant \sigma<\frac{4}{N}$, and in particular for the classical critical case $N=2, \sigma=1$. Following the modulation theory, we derive the reduced system of ordinary differential equations for the Schrödinger-Helmholtz (SH) system. We observe that the reduced equations for this $\mathrm{SH}$ system are more complicated than the equations of some other perturbation regularizations of the classical NLS equation. The detailed analysis of the reduced system on how the regularization prevents singularity formation will be presented in a forthcoming paper.
\end{abstract}

Mathematics Subject Classification: 35Q40, 35Q55

\footnotetext{
4 Also at: Department of Computer Science and Applied Mathematics, Weizmann Institute of Science, Rehovot 76100, Israel.
} 


\section{Introduction}

The nonlinear Schrödinger (NLS) equation:

$$
\begin{aligned}
& \mathrm{i} v_{t}+\Delta v+|v|^{2 \sigma} v=0, \quad x \in \mathbb{R}^{N}, \quad t \in \mathbb{R}, \\
& v(0)=v_{0},
\end{aligned}
$$

where $v$ is a complex-valued function in $\mathbb{R}^{N} \times \mathbb{R}$, arises in various physical contexts describing wave propagation in nonlinear media (see, e.g., [30,45, 46, 52]). For example, when $\sigma=1$, equation (1) describes propagation of a laser beam in a nonlinear optical medium whose index of refraction is proportional to the wave intensity. Also, the NLS equation successfully models other wave phenomena such as water waves at the free surface of an ideal fluid as well as plasma waves. In all cases, it is interesting to note that equation (1) describes wave propagation in nonhomogeneous linear media with self-induced potential given by $|v|^{2 \sigma}$.

As mentioned above, the $\sigma=1$ case is particularly interesting for laser beam propagation in optical Kerr media. Depending on the dimensionality of the space in which the beam is propagating, the wave dynamics can be either 'simple' or 'intricate'. In one space dimension, the NLS equation is known to be integrable and possesses soliton solutions that preserve their structure upon collision [1]. The picture in two dimensional (2D) space is totally different. The 2D NLS equation is not integrable, hence no exact soliton solutions are known. Instead, the 2D NLS equation admits the waveguide solution (also known as Townes soliton) $v(x, y, t)=R(r) \exp (\mathrm{i} t)$ with $r=\sqrt{x^{2}+y^{2}}$, where $R>0$ satisfies the nonlinear boundary value problem

$$
\frac{\mathrm{d}^{2} R}{\mathrm{~d} r^{2}}+\frac{1}{r} \frac{\mathrm{d} R}{\mathrm{~d} r}-R+R^{3}=0, \quad \frac{\mathrm{d} R}{\mathrm{~d} r}(0)=0, \quad \lim _{r \rightarrow+\infty} R(r)=0 .
$$

Most importantly, the $L^{2}$ norm (or power in optics) of the Townes soliton defines a critical value for blow up for the 2D critical NLS, that is, if initially the beam's power is larger than that of the Townes soliton, $\left\|v_{0}\right\|_{L^{2}}^{2}>\|R\|_{L^{2}}^{2}$, then the beam undergoes a finite time blow up. If on the other hand, $\left\|v_{0}\right\|_{L^{2}}^{2}<\|R\|_{L^{2}}^{2}$ then the wave will diffract and the solution exists globally in time. Various mechanisms to arrest the collapse have been suggested, by modifying the NLS equation, such as nonparaxiality [15] or the addition of higher order dispersion [16]. As a result, an important issue that arises in the mathematical study of the NLS is the question of local and global existence of solutions, their uniqueness as well as the profile of blow-up solutions. Knowing answers to such questions may have some consequences on possible physical observations of phenomenon governed by the NLS and in validating its derivation. In the works of Ginibre and Velo [23] and Weinstein [48], it is proved that equation (1) has a unique global solution when $0<\sigma<\frac{2}{N}$, and that it has a unique global solution for 'small' initial data for the critical case $\sigma=\frac{2}{N}$. The proof of global existence uses the fact that the energy $\mathcal{N}(v)=\int_{\mathbb{R}^{N}}|v(x, t)|^{2} \mathrm{~d} x$ and the Hamiltonian $\mathcal{H}(v)=\int_{\mathbb{R}^{N}}\left(|\nabla v(x, t)|^{2}-\frac{|v(x, t)|^{2 \sigma+2}}{\sigma+1}\right) \mathrm{d} x$ are conserved quantities of the dynamics of (1). In the case of $\sigma \geqslant \frac{2}{N}$, Glassey [24] proved that there exist solutions that develop singularities in finite time. In the critical case $\sigma=\frac{2}{N}$, Merle and coworkers got some remarkable results with numerical computation (see $[18,36,37]$ and references therein). Specifically in [18], Fibich and Merle studied self-focusing in bounded domain using a combination of rigorous, asymptotic and numerical results. In [37], Merle and Raphael obtained a sharp lower bound on the blow-up rate for the $L^{2}$ norm for critical NLS in $\mathbb{R}^{N}$.

Instead of the potential $|v|^{2 \sigma}$, some physicists consider a self-gravitational potential (see, e.g., $[41,43])$ and come to a new system: Schrödinger-Newton (SN) (Schrödinger-Poisson) 
equation:

$$
\begin{aligned}
& \mathrm{i} v_{t}+\Delta v+\psi v=0, \quad x \in \mathbb{R}^{N}, \quad t \in \mathbb{R}, \\
& -\alpha^{2} \Delta \psi=|v|^{2}, \\
& v(0)=v_{0},
\end{aligned}
$$

where $\alpha>0$ is a real constant. System (3) is a Hamiltonian system with the corresponding Hamiltonian $\mathcal{H}(v)=\int_{\mathbb{R}^{N}}\left(|\nabla v(x, t)|^{2}-\frac{\psi(x, t)|v(x, t)|^{2}}{2}\right) \mathrm{d} x$ and (3) can be obtained formally by the variational principle $\mathrm{i} \frac{\partial v}{\partial t}=\frac{\delta \mathcal{H}(v)}{\delta v^{*}}$, where $v^{*}$ denotes the complex conjugate of $v$. This coupled system of equations consists of the Schrödinger equation for a wave function $v$ moving in a potential $\psi$, where $\psi$ is obtained by solving the Poisson equation with source $\rho=|v|^{2}$. Observe that when the dimension $N=3$ in this case, the Green function of the Laplace operator is $\frac{1}{|x|}$, which results in the Hartree potential $\psi(x)=\left(\frac{1}{|x|}\right) *|v|^{2}$. Hayashi and Ozawa [26] have studied this NLS system with Hartree potential. They showed existence, uniqueness and some smoothing effects. Castella [9] also studied in $N=3$ a system of infinitely many coupled Schrödinger equations with Hartree potential, which includes Hayashi's system as a particular case. In [10], this convolution potential was generalized to any dimension $N$ with a potential of the form $\psi(x)=W *\left(|v|^{2}\right)$ for some function kernel $W$ in $L^{p}$ space for some $p$ such that $p \geqslant 1, p>\frac{4}{N}$. In the SN (Schrödinger-Poisson) system, $W$ is the Green function of the Laplace operator for any dimension $N$.

Inspired by the $\alpha$-models of turbulence (see, e.g., $[8,11,12,21,27,28]$ and references therein), we introduce a generalization of (3), the Schrödinger-Helmholtz (SH) (SchrödingerBessel) regularization of the classical NLS:

$$
\begin{aligned}
& \mathrm{i} v_{t}+\Delta v+u|v|^{\sigma-1} v=0, \quad x \in \mathbb{R}^{N}, \quad t \in \mathbb{R}, \\
& u-\alpha^{2} \Delta u=|v|^{\sigma+1}, \\
& v(0)=v_{0},
\end{aligned}
$$

where $\alpha>0$ and $\sigma \geqslant 1$. System (4) is a Hamiltonian system with the corresponding Hamiltonian $\mathcal{H}(v)=\int_{\mathbb{R}^{N}}\left(|\nabla v(x, t)|^{2}-\frac{u(x, t)|v(x, t)|^{\sigma+1}}{\sigma+1}\right) \mathrm{d} x$ and can be obtained formally by the variational principle $\mathrm{i} \frac{\partial v}{\partial t}=\frac{\delta \mathcal{H}(v)}{\delta v^{*}}$, where again $v^{*}$ denotes the complex conjugate of $v$. In this system, we can regard the wave function $v$ as moving in a potential $u|v|^{\sigma-1}$, where $u$ is obtained by solving the Helmholtz elliptic problem $u-\alpha^{2} \triangle u=|v|^{\sigma+1}$ whose Green function is the Bessel potential. Observe that the energy $\mathcal{N}(v)=\int_{\mathbb{R}^{N}}|v(x, t)|^{2} \mathrm{~d} x$ and the Hamiltonian $\mathcal{H}(v)=\int_{\mathbb{R}^{N}}\left(|\nabla v(x, t)|^{2}-\frac{u(x, t)|v(x, t)|^{\sigma+1}}{\sigma+1}\right) \mathrm{d} x$ are conserved in this system. When $\sigma=1$, we have the Hartree potential $u$ as in the $\mathrm{SN}$ with the only difference that the Poisson equation is modified as a Helmholtz equation. So we consider this system as a modification of SN. For $\alpha>0$, we can solve the second equation by $u=B *\left(|v|^{\sigma+1}\right)$ where $B$ is the Bessel potential of order 2. A more important fact is that when $\alpha=0$, one recovers the classical NLS, therefore we regard the system (4) as a regularization of the classical NLS. In this paper we focus our analytical study on the case $\alpha>0$ and observe that it is not difficult to show that the solutions of the regularized system converge, as $\alpha \rightarrow 0^{+}$, to the solution of the classical NLS when the latter exists. Motivated by this observation we will investigate, in a subsequent work, the behaviour when $\alpha \rightarrow 0^{+}$. In particular, we will investigate numerically the dynamics in the critical case $\sigma=\frac{2}{N}$ when letting the positive parameter $\alpha$ approach zero.

In this paper, we will study the question of local and global existence of a unique solution for system (4). Specifically, we will prove the short time existence of the unique solution, when $1 \leqslant \sigma<\frac{4}{N-2}$ (we define once and for all $\frac{4}{N-2}=\infty$ when $N \leqslant 2$ ). Moreover, we will show 
the global existence of unique solution when $1 \leqslant \sigma<\frac{4}{N}(N>1)$ and $1 \leqslant \sigma<3(N=1)$. The proof will follow the ideas of [23] and [48] and use the important fact of the conservation of the corresponding energy and the Hamiltonian of (4). Kato [29] extended Ginibre and Velo's work [23] to more generalized nonlinear term. However, that result applies to local composition operator only. Our system involves a nonlocal nonlinear operator. Therefore, our result does not follow from, and generalizes, the work of Kato. In particular, we prove short time existence of unique solution when $1 \leqslant \sigma<\frac{4}{N-2}$, and global existence of unique solution when $1 \leqslant \sigma<\frac{4}{N}$ for $N>1$ and $1 \leqslant \sigma<3$ for $N=1$. Comparing with the results of the classical NLS (1) $\left(\sigma<\frac{2}{N-2}\right.$ for local existence and $\sigma<\frac{2}{N}$ for global existence), one expects these 'better' results for (3) and (4) since the nonlinear terms in (3) and (4) are milder than that of the classical NLS (1). The parameter $\alpha$ plays an important role in our proofs. The Bessel potential, which is the Green function of the Helmholtz operator, regularizes the potential in the system. Instead of applying the fact that the Bessel potential merely lies in certain $L^{p}$ space and applying Young's inequality for convolution as in [10] and getting global results for only $\sigma<\frac{2}{N}$ as in classical NLS, we apply the elliptic regularization property of the Helmholtz equation through the Calderón-Zygmund singular integral regularization of the convolution with the Bessel potential ([22, 25,31,50,51] and references therein), which gives us better results.

In the last section, we use modulation theory to study the reduced system of (4), and compare the results with some other modification (regularization ) of the Schrödinger systems with perturbations ([15-17]). Indeed, we can rewrite the equation in (4) as

$$
\mathrm{i} v_{t}+\Delta v+|v|^{2 \sigma} v+\alpha^{2}(\triangle u) v=0 .
$$

When $\alpha$ is a small real parameter, we can regard the last term as a perturbation of the classical NLS. The purpose of modulation theory is to explain the role of the regularization in preventing the formation of a singularity near the critical values of the initial data which blow up in the classical critical case. In general, by reducing the time-space variable system into a system with only time variable, it is easier to perform numerical simulations and investigate the dynamics near singularity. However, we observe here that the first leading order reduced system of (4), in the classical critical case $\sigma=\frac{2}{N}$ (for any $\alpha>0$ ), is more complicated when compared with some other perturbed regularizations of the classical NLS equation [14, 19, 20, 33, 35], where the latter bear a very simple generic form and yield focusing-defocusing oscillation, which explains the prevention of singularity for those perturbations. The perturbed term in the SH system is represented in a complicated integral form, which we do not analyse here for its asymptotic behaviour near singularity formation. The detailed study of this integral is out of the scope of this paper and we will present further study of modulation theory and the reduced system in a forthcoming paper.

In section 2, we will introduce some essential notation and definitions, and some preliminary results that will be used throughout the paper. Following the work of Ginibre and Velo [23], we prove in section 3 local (in time) existence and uniqueness of solution for system (4) using the contraction mapping principle. In section 4, we will extend the local solution to global existence, for $1 \leqslant \sigma<\frac{4}{N}(N>1)$ and $1 \leqslant \sigma<3(N=1)$, after establishing the required a priori estimates for the $H^{1}$ norm of the solution, which remains finite for every finite interval of time. Note that the existence of an uniqueness result also applies to system (3) since the singular part of the Bessel potential behaves in a similar way as the Newton potential and the Calderón-Zygmund singular theory for elliptic regularity applies equally. 


\section{Notation and preliminaries}

In this section we introduce some preliminary results and the basic notation and definitions that will be used throughout this paper.

We denote by $\|\cdot\|_{p}$ the norm in the space $L^{p}=L^{p}\left(\mathbb{R}^{N}\right)(1 \leqslant p \leqslant \infty)$, except for $p=2$ where the subscript 2 will be omitted. We will denote by $\langle\cdot, \cdot\rangle$ the scalar product in $L^{2}$. The conjugate pair $p, p^{\prime}$ satisfies the relation $\frac{1}{p}+\frac{1}{p^{\prime}}=1$. For any real number $l$, we denote by $H^{l}=H^{l}\left(\mathbb{R}^{N}\right)$, the usual Sobolev space. Of special interest is the $H^{1}$ Sobolev space with the norm defined by

$$
\|v\|_{H^{1}}^{2}=\int_{\mathbb{R}^{N}}\left(1+|\xi|^{2}\right)|\hat{v}(\xi)|^{2} \mathrm{~d} \xi,
$$

or equivalently

$$
\|v\|_{H^{1}}^{2}=\|v\|^{2}+\|\nabla v\|^{2}
$$

We denote by $\|u\|_{W^{k, p}}=\left(\Sigma_{|\alpha| \leqslant k} \int_{\mathbb{R}^{N}}\left|D^{\alpha} u\right|^{p} \mathrm{~d} x\right)^{1 / p}, 1 \leqslant p<\infty$, for $u$ belonging to the Sobolev space $W^{k, p}\left(\mathbb{R}^{N}\right)$. For any interval $I$ of the real line $\mathbb{R}$, and for any Banach space $\mathcal{B}$, we denote by $\mathcal{C}(I, \mathcal{B})$ (respectively, $\mathcal{C}_{b}(I, \mathcal{B})$ ) the space of continuous (respectively, bounded continuous) functions from $I$ into $\mathcal{B}$.

In this paper $C$ and $C_{\alpha}$ will denote constants which might depend on various parameters of the problem. They might vary in value from one time to another, but they are independent of the solution. When it is relevant we will comment on the asymptotic behaviour of these constants as they depend on the corresponding parameters.

First, we recall some classical Gagliardo-Nirenberg and Sobolev inequalities (see, e.g., [6]).

\section{Proposition 1.}

(1) For any $N \geqslant 1$, we have

$$
\begin{aligned}
& \|v\|_{q} \leqslant C\|v\|^{1-\frac{q-2}{2 q} N}\|v\|_{H^{1}}^{\frac{q-2}{2 q} N} \quad \text { for every } v \in H^{1}, 0<\frac{q-2}{2 q} N \leqslant 1, \\
& \|v\|_{q} \leqslant C\|v\|_{W^{2, p}} \quad \text { for every } v \in W^{2, p}, q \geqslant p, \quad 2 p>N, \\
& \|v\|_{q} \leqslant C\|v\|_{W^{2, p}} \quad \text { for every } v \in W^{2, p}, \frac{1}{q} \geqslant \frac{1}{p}-\frac{2}{N} \geqslant 0, \quad q<\infty .
\end{aligned}
$$

In particular,

$$
\|v\|_{q} \leqslant C\|v\|_{W^{2,2}}=C\|v\|_{H^{2}} \quad \text { for every } v \in H^{2}, 2 \leqslant q \leqslant \infty, \quad N \leqslant 3 .
$$

(2) $\operatorname{For} N=2$,

$$
\|v\|_{q} \leqslant C\|v\|_{H^{1}} \quad \text { for every } v \in H^{1}, 2 \leqslant q<\infty .
$$

(3) $\operatorname{For} N=1$,

$$
\|v\|_{q} \leqslant C\|v\|_{H^{1}} \quad \text { for every } v \in H^{1}, 2 \leqslant q \leqslant \infty .
$$

With these inequalities at hand, we can process the nonlinear term. Let us rewrite the nonlinear term

$$
f(v)=u|v|^{\sigma-1} v=B\left(|v|^{\sigma+1}\right)|v|^{\sigma-1} v,
$$

where $B=\left(I-\alpha^{2} \triangle\right)^{-1}$, the inverse of the Helmholtz operator. Then $f$ is a locally Lipschitz mapping from $H^{1}$ into $L^{r^{\prime}}$, for some $r \in\left(2, \frac{2 N}{N-2}\right]$, where $\frac{1}{r}+\frac{1}{r^{\prime}}=1$. 
Proposition 2. Let $N \geqslant 1$ and $1 \leqslant \sigma<\frac{4}{N-2}$. For every $v_{1}, v_{2} \in H^{1} \subset L^{r}$, where $r$ depends on the given $\sigma$ and belongs to the range $r \in\left[2, \frac{2 N}{N-2}\right.$ ) (we consider $\frac{2 N}{N-2}$ as $\infty$ when $N \leqslant 2$ ), we have $\left\|f\left(v_{1}\right)-f\left(v_{2}\right)\right\|_{r^{\prime}} \leqslant k\left\|v_{1}-v_{2}\right\|_{r}$, where $k=C_{\alpha, \sigma}\left(\left\|v_{1}\right\|_{H^{1}}+\left\|v_{2}\right\|_{H^{1}}\right)^{2 \sigma}$ and $\frac{1}{r}+\frac{1}{r^{\prime}}=1$, for some constant $C_{\alpha, \sigma}$.

Before we prove this proposition, we will state the following lemmas:

Lemma 3. Let $N \geqslant 1$, for any $0<\sigma<\frac{4}{N-2}$, there exists $r \in\left[2, \frac{2 N}{N-2}\right)$, which depends on $\sigma$, such that for every $v_{1}, v_{2}, v \in H^{1} \subset L^{r}$, we have

$$
\left\|B\left(|v|^{\sigma}\left|v_{1}-v_{2}\right|\right)|v|^{\sigma}\right\|_{r^{\prime}} \leqslant C_{\alpha}\|v\|_{H^{1}}^{2 \sigma}\left\|v_{1}-v_{2}\right\|_{r} .
$$

Proof. First, denote

$$
I_{1}=\left\|B\left(|v|^{\sigma}\left|v_{1}-v_{2}\right|\right)|v|^{\sigma}\right\|_{r^{\prime}}
$$

By Hölder's inequality, we have

$$
I_{1} \leqslant\left\|B\left(|v|^{\sigma}\left|v_{1}-v_{2}\right|\right)\right\|_{r^{\prime} \beta_{1}}\left\||v|^{\sigma}\right\|_{r^{\prime} \gamma_{1}},
$$

where $\beta_{1}, \gamma_{1}$ are a conjugate pair, i.e. $\frac{1}{\beta_{1}}+\frac{1}{\gamma_{1}}=1, \beta_{1}, \gamma_{1}>1$.

Now, for the elliptic equation

$$
\phi(x)-\alpha^{2} \triangle \phi(x)=\psi(x), \quad x \in \mathbb{R}^{N},
$$

we have the regularity property $[39,44,50,51]$

$$
\|\phi\|_{W^{2, p}} \leqslant C_{\alpha}\|\psi\|_{p} \quad \text { for any } 1<p<\infty,
$$

where $C_{\alpha}$ depends on $N, p$ and $\alpha$, and $C_{\alpha} \sim \frac{1}{\alpha^{2}}$ as $\alpha \rightarrow 0^{+}$. Moreover, for $\alpha$ fixed, $C_{\alpha} \sim p$ as $p \rightarrow \infty$.

Let $p>1$ to be determined later, by (9) or (10), we have

$$
\left\|B\left(|v|^{\sigma}\left|v_{1}-v_{2}\right|\right)\right\|_{r^{\prime} \beta_{1}} \leqslant C\left\|B\left(|v|^{\sigma}\left|v_{1}-v_{2}\right|\right)\right\|_{W^{2, p}}
$$

provided $\frac{1}{r^{\prime} \beta_{1}} \geqslant \frac{1}{p}-\frac{2}{N}$ if $\frac{1}{p}-\frac{2}{N} \geqslant 0$, or $r^{\prime} \beta_{1} \geqslant p$ if $\frac{1}{p}-\frac{2}{N}<0$.

Applying the elliptic regularity property (18) and Hölder's inequality to the above term, we get

$$
\begin{aligned}
\left\|B\left(|v|^{\sigma}\left|v_{1}-v_{2}\right|\right)\right\|_{r^{\prime} \beta_{1}} & \leqslant C_{\alpha}\left\||v|^{\sigma}\right\| v_{1}-v_{2} \mid \|_{p}, \\
& \leqslant C_{\alpha}\|v\|_{p \theta^{\prime} \sigma}^{\sigma}\left\|v_{1}-v_{2}\right\|_{p \theta},
\end{aligned}
$$

where $\frac{1}{\theta}+\frac{1}{\theta^{\prime}}=1, \theta, \theta^{\prime}>1$.

Therefore, we obtain

$$
I_{1} \leqslant C_{\alpha}\|v\|_{p \theta \sigma}^{\sigma}\|v\|_{r^{\prime} \gamma_{1} \sigma}^{\sigma}\left\|v_{1}-v_{2}\right\|_{p \theta^{\prime}}
$$

Now, by requiring $p \theta^{\prime}=r, p \theta \sigma=r^{\prime} \gamma_{1} \sigma=r$, we have

$$
\begin{aligned}
& \theta^{\prime}=\frac{r}{p}, \\
& p=\frac{r}{\sigma+1} \in(1, r) \Rightarrow 0<\sigma<r-1, \\
& \beta_{1}=\frac{r-1}{(r-1)-\sigma}>1 \Rightarrow \sigma<r-1, \\
& r^{\prime} \beta_{1} \geqslant p \Rightarrow \sigma \geqslant \frac{r-2}{2} \quad \text { or } \quad \frac{1}{r^{\prime} \beta_{1}} \geqslant \frac{1}{p}-\frac{2}{N} \Rightarrow \sigma \leqslant \frac{N+2}{2 N} r-1 .
\end{aligned}
$$

So for given $0<\sigma<\frac{4}{N-2}$, we can always find an $r \in\left[2, \frac{2 N}{N-2}\right.$ ) satisfying all the conditions above, then we have

$$
I_{1} \leqslant C_{\alpha}\|v\|_{H^{1}}^{2 \sigma}\left\|v_{1}-v_{2}\right\|_{r}
$$


Lemma 4. Let $N \geqslant 1$, for $1 \leqslant \sigma<\frac{4}{N-2}$, there exists $r \in\left[2, \frac{2 N}{N-2}\right)$, which depends on $\sigma$, such that for every $v_{1}, v_{2}, v \in H^{1} \subset L^{r}$, we have

$$
\left\|B\left(|v|^{\sigma+1}\right)|v|^{\sigma-1}\left(v_{1}-v_{2}\right)\right\|_{r^{\prime}} \leqslant C_{\alpha}\|v\|_{H^{1}}^{2 \sigma}\left\|v_{1}-v_{2}\right\|_{r} .
$$

Proof. Denote

$$
I_{2}=\left\|B\left(|v|^{\sigma+1}\right)|v|^{\sigma-1}\left(v_{1}-v_{2}\right)\right\|_{r^{\prime}} .
$$

By Hölder's inequality, we have

$$
\begin{aligned}
I_{2} & \leqslant\left\|B\left(|v|^{\sigma+1}\right)\right\|_{r^{\prime} \theta_{1}}\left\||v|^{\sigma-1}\right\|_{r^{\prime} \beta_{1}}\left\|v_{1}-v_{2}\right\|_{r^{\prime} \gamma_{1}}, \\
& =\left\|B\left(|v|^{\sigma+1}\right)\right\|_{r^{\prime} \theta_{1}}\|v\|_{(\sigma-1) r^{\prime} \beta_{1}}^{\sigma-1}\left\|v_{1}-v_{2}\right\|_{r^{\prime} \gamma_{1}},
\end{aligned}
$$

where $\frac{1}{\theta_{1}}+\frac{1}{\beta_{1}}+\frac{1}{\gamma_{1}}=1$.

Letting $p>1$ to be determined later, by (9) or (10), we have

$$
\left\|B\left(|v|^{\sigma+1}\right)\right\|_{r^{\prime} \theta_{1}} \leqslant C\left\|B\left(|v|^{\sigma+1}\right)\right\|_{W^{2, p}}
$$

provided $\frac{1}{r^{\prime} \theta_{1}} \geqslant \frac{1}{p}-\frac{2}{N}$ if $\frac{1}{p}-\frac{2}{N} \geqslant 0$, or $r^{\prime} \theta_{1} \geqslant p$ if $\frac{1}{p}-\frac{2}{N}<0$.

Applying the elliptic regularity property (18) and Hölder's inequality to the above term, we get

$$
\begin{aligned}
\left\|B\left(|v|^{\sigma+1}\right)\right\|_{r^{\prime} \theta_{1}} & \leqslant C_{\alpha}\left\||v|^{\sigma+1}\right\|_{p}, \\
& =C_{\alpha}\|v\|_{(\sigma+1) p}^{\sigma+1} .
\end{aligned}
$$

Then we obtain

$$
I_{2} \leqslant C_{\alpha}\|v\|_{(\sigma+1) p}^{\sigma+1}\|v\|_{(\sigma-1) r^{\prime} \beta_{1}}^{\sigma-1}\left\|v_{1}-v_{2}\right\|_{r^{\prime} \gamma_{1}} .
$$

Now, by requiring $(\sigma+1) p=(\sigma-1) r^{\prime} \beta_{1}=r^{\prime} \gamma_{1}=r$ (choose $\beta_{1}=\infty$ when $\left.\sigma=1\right)$, we have

$$
\begin{aligned}
& \theta_{1}=\frac{r-1}{r-1-\sigma}>1 \Rightarrow \sigma<r-1, \\
& \beta_{1}=\frac{r-1}{\sigma-1}>1 \Rightarrow \sigma<r, \\
& \gamma_{1}=r-1>1, \\
& p=\frac{r}{\sigma+1}>1 \Rightarrow \sigma<r-1 \\
& \sigma \leqslant \frac{N+2}{2 N} r-1 \quad \text { or } \quad \sigma \geqslant \frac{r-2}{2} .
\end{aligned}
$$

So for $1 \leqslant \sigma<\frac{4}{N-2}$, we can always find an $r \in\left[2, \frac{2 N}{N-2}\right)$ satisfying all the conditions above, then

$$
I_{2} \leqslant C_{\alpha}\|v\|_{H^{1}}^{2 \sigma}\left\|v_{1}-v_{2}\right\|_{r}
$$

Now, we are ready to prove proposition 2 .

Proof. Recall that $f(v)=B\left(|v|^{\sigma+1}\right)|v|^{\sigma-1} v$, by triangle inequality, we have

$$
\begin{aligned}
\left\|f\left(v_{1}\right)-f\left(v_{2}\right)\right\|_{r^{\prime}} & \left.=\| B\left(\left|v_{1}\right|^{\sigma+1}\right)\left|v_{1}\right|^{\sigma-1} v_{1}\right)-B\left(\left|v_{2}\right|^{\sigma+1}\right)\left|v_{2}\right|^{\sigma-1} v_{2} \|_{r^{\prime}} \\
\leqslant & \|\left(B \left(\left|v_{1}\right|^{\sigma+1}-B\left(\left|v_{2}\right|^{\sigma+1}\right)\left|v_{2}\right|^{\sigma-1} v_{2} \|_{r^{\prime}}\right.\right. \\
& +\left\|B\left(\left|v_{1}\right|^{\sigma+1}\right)\left(\left|v_{1}\right|^{\sigma-1} v_{1}-\left|v_{2}\right|^{\sigma-1} v_{2}\right)\right\|_{r^{\prime}} .
\end{aligned}
$$


Denote

$$
\begin{aligned}
& I_{1}=\|\left(B \left(\left|v_{1}\right|^{\sigma+1}-B\left(\left|v_{2}\right|^{\sigma+1}\right)\left|v_{2}\right|^{\sigma-1} v_{2} \|_{r^{\prime}},\right.\right. \\
& I_{2}=\left\|B\left(\left|v_{1}\right|^{\sigma+1}\right)\left(\left|v_{1}\right|^{\sigma-1} v_{1}-\left|v_{2}\right|^{\sigma-1} v_{2}\right)\right\|_{r^{\prime}} .
\end{aligned}
$$

Let us consider $I_{1}$ first.

Since

$$
\left.|| v_{1}\right|^{\sigma+1}-\left|v_{2}\right|^{\sigma+1}\left|\leqslant C_{\sigma}\left(\left|v_{1}\right|+\left|v_{2}\right|\right)^{\sigma}\right| v_{1}-v_{2} \mid,
$$

where $C_{\sigma}$ is some constant depending on $\sigma$, applying the comparison principle to the elliptic equation (17), we have the following monotonicity property for the operator $B=\left(I-\alpha^{2} \triangle\right)^{-1}$ :

$$
|B(\phi)| \leqslant B(|\phi|) \quad \text { for complex-valued function } \phi .
$$

where $|\cdot|$ denotes the absolute value (modulus) of a complex value.

Now by linearity of the operator $B$, we have

$$
\begin{aligned}
\left|B\left(\left|v_{1}\right|^{\sigma+1}\right)-B\left(\left|v_{2}\right|^{\sigma+1}\right)\right| & =\left|B\left(\left|v_{1}\right|^{\sigma+1}-\left|v_{2}\right|^{\sigma+1}\right)\right|, \\
& \leqslant B\left(\left.|| v_{1}\right|^{\sigma+1}-\left|v_{2}\right|^{\sigma+1} \mid\right), \\
& \leqslant C_{\sigma} B\left(\left(\left|v_{1}\right|+\left|v_{2}\right|\right)^{\sigma}\left|v_{1}-v_{2}\right|\right) .
\end{aligned}
$$

Plugging this result into $I_{1}$ and applying lemma 3 , we get

$$
\begin{aligned}
I_{1} & \leqslant C_{\sigma}\left\|B\left(\left(\left|v_{1}\right|+\left|v_{2}\right|\right)^{\sigma}\left|v_{1}-v_{2}\right|\right)\left|v_{2}\right|^{\sigma-1} v_{2}\right\|_{r^{\prime}}, \\
& \leqslant C_{\sigma}\left\|B\left(\left(\left|v_{1}\right|+\left|v_{2}\right|\right)^{\sigma}\left|v_{1}-v_{2}\right|\right)\left(\left|v_{1}\right|+\left|v_{2}\right|\right)^{\sigma}\right\|_{r^{\prime}}, \\
& \leqslant C_{\alpha, \sigma}\left(\left\|v_{1}\right\|_{H^{1}}+\left\|v_{2}\right\|_{H^{1}}\right)^{2 \sigma}\left\|v_{1}-v_{2}\right\|_{r} .
\end{aligned}
$$

Now we consider $I_{2}$.

Since

$$
\left.|| v_{1}\right|^{\sigma-1} v_{1}-\left|v_{2}\right|^{\sigma-1} v_{2}\left|\leqslant C_{\sigma}\left(\left|v_{1}\right|+\left|v_{2}\right|\right)^{\sigma-1}\right| v_{1}-v_{2} \mid,
$$

plugging the above result into $I_{2}$ and applying the comparison principle (23), we get

$$
\begin{aligned}
I_{2} & \leqslant C_{\sigma}\left\|B\left(\left|v_{1}\right|^{\sigma+1}\right)\left(\left|v_{1}\right|+\left|v_{2}\right|\right)^{\sigma-1}\left|v_{1}-v_{2}\right|\right\|_{r^{\prime}}, \\
& \leqslant C_{\sigma}\left\|B\left(\left(\left|v_{1}\right|+\left|v_{2}\right|\right)^{\sigma+1}\right)\left(\left|v_{1}\right|+\left|v_{2}\right|\right)^{\sigma-1}\left|v_{1}-v_{2}\right|\right\|_{r^{\prime}} .
\end{aligned}
$$

By lemma 4, we have

$$
I_{2} \leqslant C_{\alpha, \sigma}\left(\left\|v_{1}\right\|_{H^{1}}+\left\|v_{2}\right\|_{H^{1}}\right)^{2 \sigma}\left\|v_{1}-v_{2}\right\|_{r}
$$

Next, we will give some elementary properties of the free evolution (linear Schrödinger equation) formally defined by the group of operators

$$
U(t)=\exp (\mathrm{i} t \triangle)
$$

where $t \in \mathbb{R}$. In the following, we will state some well-known results about the operator $U(t)$ without proving them (see, e.g., $[23,45])$.

Lemma 5. For any $r \geqslant 2$, and for any $t \neq 0, U(t)$ is a bounded linear operator from $L^{r^{\prime}}$ to $L^{r}$, and the map $t \rightarrow U(t)$ is strongly continuous. Moreover, for all $t \in \mathbb{R} \backslash\{0\}$, one has

$$
\|U(t) v\|_{r} \leqslant(4 \pi|t|)^{\frac{N}{r}-\frac{N}{2}}\|v\|_{r^{\prime}}
$$

for all $v \in L^{r^{\prime}}$.

Corollary 6. Let $I$ be an interval of $\mathbb{R}$, and let $v \in \mathbb{C}\left(I, L^{r^{\prime}}\right)$. Then for all $t \in \mathbb{R}$ the map $\tau \rightarrow U(t-\tau) v(\tau)$ is continuous from $I \backslash\{t\}$ into $L^{r}$. 


\section{Short time existence and uniqueness}

In this section we will prove a local existence and uniqueness theorem of solutions to system (4) by a fixed point technique.

The integral equation

$$
v(t)=U\left(t-t_{0}\right) v_{0}+\mathrm{i} \int_{t_{0}}^{t} U(t-\tau) f(v(\tau)) \mathrm{d} \tau
$$

may be considered as the integral version of the initial value problem for equation (4).

Defining the subspace $Y(I) \subset \mathcal{C}(I, X)$ and $Y_{b}(I) \subset \mathcal{C}_{b}(I, X)$ by

$$
\begin{aligned}
& Y(I)=\{v: v \in \mathcal{C}(I, X) \text { and } v(t)=U(t-s) v(s) \text { for all } s \text { and } t \in I\}, \\
& Y_{b}(I)=Y(I) \cap \mathcal{C}_{b}(I, X) .
\end{aligned}
$$

Here for special interest we choose the Banach space $X=L^{r}\left(\mathbb{R}^{N}\right)$, for some $r>2$, which is specified in the proof of lemmas 3 and 4 , and $\bar{X}=L^{r^{\prime}}\left(\mathbb{R}^{N}\right)$.

If $v \in \mathcal{C}_{b}(I, X)$, we shall denote its norm by $|v|_{I}$, and for $v \in \mathcal{C}_{b}\left(I, H^{1}\right)$, we denote its norm by $|v|_{H^{1}, I}$. The ball of radius $R$ in $\mathcal{C}_{b}(I, X)$ will be denoted by $B(I, R)$.

Let $t_{1}, t_{2} \in \mathbb{R}$ and let $v(t)$ be a family of complex-valued functions defined on $\mathbb{R}^{N}$, depending on a parameter $t \in \mathbb{R}$. We formally define the operators

$$
\left[G\left(t_{1}, t_{2}\right) v\right](t)=\mathrm{i} \int_{t_{1}}^{t_{2}} U(t-\tau) f(v(\tau)) \mathrm{d} \tau
$$

where $f$ is the nonlinear term defined in (14). The first lemma below gives a meaning to the expression defined by (27) and contains some of its properties.

Lemma 7. For any interval $I \subset \mathbb{R}$ (possibly unbounded), the maps $\left(t_{1}, t_{2}, v\right) \rightarrow G\left(t_{1}, t_{2}\right) v$ are continuous from $I \times I \times \mathcal{C}(I, X)$ to $Y_{b}(\mathbb{R})$. Moreover, for any $t_{1}, t_{2} \in I\left(t_{1}<t_{2}\right)$, for any compact sub-interval $J$ such that $\left[t_{1}, t_{2}\right] \subset J \subset I$, and for any $t \in\left[t_{1}, t_{2}\right]$, for any $v_{1}, v_{2} \in \mathcal{C}(I, X)$ the $G$ operator satisfies the estimates

$$
\left\|G\left(t_{1}, t_{2}\right) v_{1}(t)-G\left(t_{1}, t_{2}\right) v_{2}(t)\right\|_{r} \leqslant k^{\prime}\left\|v_{1}-v_{2}\right\|_{J}\left|t_{2}-t_{1}\right|^{\frac{N}{r}-\frac{N}{2}+1},
$$

where $k^{\prime}=k(4 \pi)^{\frac{N}{r}-\frac{N}{2}}, k=C_{\alpha}\left(\left\|v_{1}\right\|_{H^{1}}+\left\|v_{2}\right\|_{H^{1}}\right)$, which is derived in the proof of proposition 2 .

Proof. For any $v \in \mathcal{C}(I, X)$ the function $\tau \rightarrow f(v(\tau))$ belongs to $\mathcal{C}(I, \bar{X})$ as a consequence of proposition 2. Therefore, by lemma 3 , for any $t \in \mathbb{R} \backslash\{t\}$ the function

$$
\tau \rightarrow U(t-\tau) f(v(\tau))
$$

is continuous from $I$ to $X$. To check the integrability of function (28) it will be enough to show the integrability of its norm. More generally one is interested in the integrability of

$$
\left\|U(t-\tau)\left[f\left(v_{1}(\tau)\right)-f\left(v_{2}(\tau)\right)\right]\right\|_{r},
$$

for any $v_{1}, v_{2} \in \mathcal{C}\left(I, H^{1}\right) \subset \mathcal{C}(I, X)$.

This is a direct consequence of proposition 2 and lemma 3 : for $t \in \mathbb{R}$, for every compact sub-interval $J \subset I$ and $\tau \in J$, we have

$$
\left\|U(t-\tau)\left[f\left(v_{1}(\tau)\right)-f\left(v_{2}(\tau)\right)\right]\right\|_{r^{\prime}} \leqslant(4 \pi|t-\tau|)^{\frac{N}{r}-\frac{N}{2}} k\left\|v_{1}-v_{2}\right\|_{J} .
$$

Finally, we come to the conclusion that

$$
\left\|G\left(t_{1}, t_{2}\right) v_{1}(t)-G\left(t_{1}, t_{2}\right) v_{2}(t)\right\| \leqslant k^{\prime}\left\|v_{1}-v_{2}\right\|_{J}\left|t_{2}-t_{1}\right|^{\frac{N}{r}-\frac{N}{2}+1} .
$$


Now, in order to study equation (26) one needs the operators

$$
\left[F\left(t_{0}\right) v\right](t)=\left[G\left(t_{0}, t\right) v\right](t) .
$$

The existence and properties of $F$ follow immediately from lemma 5.

For every $v \in \mathcal{C}\left(I, H^{1}\right) \subset \mathcal{C}(I, X)$,

$$
\left[A\left(t_{0}, v_{0}\right) v\right](t)=\left[F\left(t_{0}\right) v\right](t)+U\left(t-t_{0}\right) v_{0}
$$

is a continuous map from $\mathcal{C}\left(I, H^{1}\right) \subset \mathcal{C}(I, X)$ into $\mathcal{C}(I, X)$.

With this notation equation (26) may be rewritten as

$$
A\left(t_{0}, v_{0}\right) v=v .
$$

The following lemma gives some elementary properties of the solutions of equation (26). In particular, it expresses the consistency of the change of the initial time $t_{0}$.

Lemma 8. Let $I$ and $J$ be two intervals of $\mathbb{R}, J \subset I$, let $t_{0} \in J$, let $v_{0} \in H^{1}$ be such that the function $t \rightarrow U\left(t-t_{0}\right) v_{0}$ belongs to $Y(I)$, and let $v \in \mathcal{C}(J, X)$ be a solution of equation (32).

(i) The function

$$
\phi(v): s \rightarrow U(\cdot-s) v(s)=[\phi(v)](s)
$$

belongs to $\mathcal{C}(J, Y(I))$ and satisfies for all $s, s^{\prime} \in J$ the equality

$$
[\phi(v)](s)-[\phi(v)]\left(s^{\prime}\right)=G\left(s^{\prime}, s\right) v .
$$

Furthermore, if for some $s \in J,[\phi(v)](s) \in Y_{b}(I)$, then $\phi(v) \in \mathcal{C}\left(J, Y_{b}(I)\right)$. If in addition $J$ is bounded, then $\phi(v) \in \mathcal{C}_{b}\left(J, Y_{b}(I)\right)$.

(ii) For any $s \in J$, $u$ satisfies the equation

$$
A(s, v(s)) v=v .
$$

Proof. Applying the operator $U(t-s)$ to equation $\left[A\left(t_{0}, v_{0}\right) v\right](s)=v(s)$ and using the fact that $U(t-s)\left[G\left(t_{1}, t_{2}\right) v\right](s)=\left[G\left(t_{1}, t_{2}\right) v\right](t)$ (for the proof of this identity, we refer to Ginibre and Velo [23]) yields

$$
[\phi(v)](s)=U\left(\cdot-t_{0}\right) v_{0}+G\left(t_{0}, s\right) v .
$$

From which (34) follows immediately. The continuity properties of the left-hand side of (36) are then a consequence of the assumptions made on $v_{0}$ and of lemma 7 . Finally, putting $s^{\prime}=t$ in (34) and taking the values of both members at $t$ one obtains equation (35) at time $t$.

We are now ready to discuss the problem of the existence and uniqueness of solutions of equation (32).

Theorem 9. For any $\rho>0$, there exists a $T_{0}(\rho)>0$, depending only on $\rho$, such that for any $t_{0} \in \mathbb{R}$ and for any $v_{0} \in H^{1}$, for which $\left\|v_{0}\right\|_{H^{1}} \leqslant \rho$, equation (32) has a unique solution on $\mathcal{C}(I, X)$, where $I=\left[t_{0}-T_{0}(\rho), t_{0}+T_{0}(\rho)\right]$ and $X=L^{r}$.

Proof. Let $\rho$ be a fixed positive number, let $t_{0}$ and $T_{0} \in \mathbb{R}, T_{0}>0$, and let $I=\left[t_{0}-T_{0}, t_{0}+T_{0}\right]$. Then for every $v_{1}, v_{2} \in H^{1}$ and $\left\|v_{1}-v_{2}\right\|_{H^{1}} \leqslant 2 \rho$, lemma 5 and (30) yield the inequality

$$
\left|F\left(t_{0}\right) v_{1}-F\left(t_{0}\right) v_{2}\right|_{I} \leqslant 2 k^{\prime}\left|t-t_{0}\right|^{\frac{N}{r}-\frac{N}{2}+1}\left|v_{1}-v_{2}\right|_{I} .
$$

In particular, if we take $T=T_{0}(\rho)$ with $T_{0}(\rho)$ defined by

$$
4 k^{\prime}\left|T_{0}(\rho)-t_{0}\right|^{\frac{N}{r}-\frac{N}{2}+1}=1
$$


in equality (37) it gives

$$
\left|F\left(t_{0}\right) v_{1}-F\left(t_{0}\right) v_{2}\right|_{I} \leqslant \frac{1}{2}\left|v_{1}-v_{2}\right|_{I} .
$$

Now let $v_{0} \in X$ be such that $U\left(\cdot-t_{0}\right) v_{0} \in B(I, \rho)$. Definition (31) and estimate (39) imply

$$
\left|A\left(t_{0}, v_{0}\right) v\right|_{I} \leqslant 2 \rho
$$

and

$$
\left|A\left(t_{0}, v_{0}\right) v_{1}-A\left(t_{0}, v_{0}\right) v_{2}\right|_{I} \leqslant \frac{1}{2}\left|v_{1}-v_{2}\right|_{I},
$$

for all $v, v_{1}, v_{2} \in B(I, 2 \rho)$, from which it follows that $A\left(t_{0}, v_{0}\right)$ is a contraction from the ball $B(I, 2 \rho)$ into itself. The result is now a consequence of the contraction mapping theorem.

\section{Global in time existence of solutions}

In this section we will study global existence of solutions to system (4) under the condition of $\sigma \geqslant 1$. We will show below that we have global in time solutions when $1 \leqslant \sigma<\frac{4}{N}(N>1)$ and $1 \leqslant \sigma<3(N=1)$. Comparing this with the results of the classical $\operatorname{NLS}\left(\sigma<\frac{2}{N}\right)$, we 'gain' global regularity for a larger range of values of $\sigma$. In the case of $N=1$, we have $1 \leqslant \sigma<3$, which is a smaller range, as one expects $\frac{4}{N}$, but we still gain a larger range compared with the classical case $\sigma<2$. As we stated in the introduction, system (4) will recover the classical NLS as the parameter $\alpha \rightarrow 0^{+}$; in a subsequent paper, we will study numerically the blow-up profile of the classical NLS by focusing on system (4) with $\frac{2}{N} \leqslant \sigma<\frac{4}{N}$ when $N \leqslant 3$ especially in dimension $N=2$. To be more specific, we will compute $\mathrm{SH}$ system (4) and try to find the blow up profile by forcing the parameter $\alpha$ to approach zero.

Theorem 10. Let $v_{0} \in H^{1}\left(\mathbb{R}^{N}\right)$. If $1 \leqslant \sigma<\frac{4}{N}$ when $N>1$ and $1 \leqslant \sigma<3$ when $N=1$, then there exists a unique solution $v \in C\left((-\infty, \infty) ; H^{1}\left(\mathbb{R}^{N}\right)\right)$ of the initial value problem (4), in the sense of the equivalent integral equation.

Furthermore, as long as $v(x, t)$ remains in $H^{1}\left(\mathbb{R}^{N}\right)$, the energy

$$
\mathcal{N}(v)=\int_{\mathbb{R}^{N}}|v(x, t)|^{2} \mathrm{~d} x
$$

and Hamiltonian

$$
\mathcal{H}(v)=\int_{\mathbb{R}^{N}}\left(|\nabla v(x, t)|^{2}-\frac{u(x, t)|v(x, t)|^{\sigma+1}}{\sigma+1}\right) \mathrm{d} x
$$

remain constant in time.

In the short time existence theorem in section 3 , we have shown that the length $T_{0}$, of the interval of existence $\left[t_{0}, t_{0}+T_{0}\right]$, can be taken to depend only on $\left\|v\left(t_{0}\right)\right\|_{H^{1}}$. It follows that if $v(x, t)$ is a maximally defined solution on $\left[t_{0}, T_{\max }\right)$, then either

$$
T_{\max }=+\infty
$$

or

$$
\lim _{t \rightarrow T_{\max }^{-}}\|v(t)\|_{H^{1}}=+\infty .
$$

The heart of the global existence proof lies in the use of the invariants (42) and (43), which enable us to obtain an a priori bound of the following type:

$$
\|v(x, t)\|_{H^{1}} \leqslant C(\mathcal{N}, \mathcal{H}) .
$$


Proof. We proceed as follows:

From (43) we have

$$
\|\nabla v(x, t)\|^{2} \leqslant \mathcal{H}+\frac{1}{\sigma+1} \int_{\mathbb{R}^{N}} u|v|^{\sigma+1} \mathrm{~d} x .
$$

Observe that

$$
\begin{aligned}
\left.\left|\int_{\mathbb{R}^{N}} u\right| v\right|^{\sigma+1} \mathrm{~d} x \mid & \leqslant\|u\|_{p}\left\||v|^{\sigma+1}\right\|_{p^{\prime}} \\
& =\|u\|_{p}\|v\|_{p^{\prime}(\sigma+1)}^{\sigma+1},
\end{aligned}
$$

where

$$
\frac{1}{p}+\frac{1}{p^{\prime}}=1, \quad 1 \leqslant p, \quad p^{\prime} \leqslant \infty
$$

Case 1. $N=1$ : taking $p=\infty, p^{\prime}=1$ in the above inequality, by (13), we get

$$
\begin{aligned}
\left.\left|\int_{\mathbb{R}^{N}} u\right| v\right|^{\sigma+1} \mathrm{~d} x \mid & \leqslant\|u\|_{\infty}\|v\|_{\sigma+1}^{\sigma+1}, \\
& \leqslant C\|u\|_{H^{1}}\|v\|_{\sigma+1}^{\sigma+1} .
\end{aligned}
$$

On the other hand,

$$
\begin{aligned}
\left.\left|\int_{\mathbb{R}^{N}} u\right| v\right|^{\sigma+1} \mathrm{~d} x \mid & =\left|\int_{\mathbb{R}^{N}} u\left(u-\alpha^{2} \triangle u\right) \mathrm{d} x\right|, \\
& =\left.\left|\int_{\mathbb{R}^{N}} u^{2}+\alpha^{2}\right| \nabla u\right|^{2} \mathrm{~d} x \mid, \\
& \geqslant C_{\alpha}^{\prime}\|u\|_{H^{1}}^{2},
\end{aligned}
$$

where $C_{\alpha}^{\prime}=\min \left\{1, \alpha^{2}\right\}$.

From (47) and (48), we get

$$
\|u\|_{H^{1}} \leqslant \frac{C}{C_{\alpha}^{\prime}}\|v\|_{\sigma+1}^{\sigma+1} .
$$

Then

$$
\begin{aligned}
\left.\left|\int_{\mathbb{R}^{N}} u\right| v\right|^{\sigma+1} \mathrm{~d} x \mid & \leqslant \max \left\{1, \alpha^{2}\right\}\|u\|_{H^{1}}^{2}, \\
& \leqslant C_{\alpha}\|v\|_{\sigma+1}^{2(\sigma+1)},
\end{aligned}
$$

where $C_{\alpha}=C \max \left\{1, \alpha^{2}\right\} / \min \left\{1, \alpha^{2}\right\} \sim \frac{1}{\alpha^{2}}$ when $\alpha$ is very small.

When $\sigma=1$, the term on the right-hand side is bounded due to conservation of energy $\mathcal{N}$. Now we consider $\sigma>1$, by (8), taking $q=\sigma+1$, we obtain

$$
\|v\|_{\sigma+1} \leqslant C\|v\|_{H^{1}}^{\frac{(\sigma+1)-2}{2(\sigma+1)}}\|v\|^{1-\frac{(\sigma+1)-2}{2(\sigma+1)}},
$$

where $0<\frac{(\sigma+1)-2}{2(\sigma+1)}<1$ is always satisfied.

Plugging the above inequality into (49), we obtain

$$
\left.\left|\int_{\mathbb{R}^{N}} u\right| v\right|^{\sigma+1} \mathrm{~d} x \mid \leqslant C_{\alpha}\|v\|^{\sigma+3}\|v\|_{H^{1}}^{\sigma-1} .
$$

Then (45) yields

$$
\|v\|_{H^{1}}^{2}=\|\nabla v\|^{2}+\|v\|^{2} \leqslant \mathcal{H}_{0}+\mathcal{N}_{0}+C_{\alpha}\left\|v_{0}\right\|^{\sigma+3}\|v\|_{H^{1}}^{\sigma-1} .
$$


For (51), $\|v\|_{H^{1}}$ is uniformly bounded when $\sigma-1<2$, i.e. $\sigma<3$. So we have global existence for $1 \leqslant \sigma<3$ in the case $N=1$.

Case 2. $N=2$ : taking $2 \leqslant p<\infty$ in (46) and applying (12), we get

$$
\begin{aligned}
\left.\left|\int_{\mathbb{R}^{N}} u\right| v\right|^{\sigma+1} \mathrm{~d} x \mid & \leqslant\|u\|_{p}\|v\|_{p^{\prime}(\sigma+1)}^{\sigma+1}, \\
& \leqslant C\|u\|_{H^{1}}\|v\|_{p^{\prime}(\sigma+1)}^{\sigma+1} .
\end{aligned}
$$

Now apply the same calculation as in (48) and (49), since

$$
\min \left\{1, \alpha^{2}\right\}\|u\|_{H^{1}} \leqslant \int_{\mathbb{R}^{N}} u^{2}+\alpha^{2}|\nabla u|^{2} \mathrm{~d} x=\left|\int_{\mathbb{R}^{N}} u v^{\sigma+1} \mathrm{~d} x\right| \leqslant \max \left\{1, \alpha^{2}\right\}\|u\|_{H^{1}}^{2} .
$$

Then we have

$$
\begin{aligned}
\left|\int_{\mathbb{R}^{N}} u v^{\sigma+1} \mathrm{~d} x\right| & =\|u\|_{H^{1}(\alpha)}^{2}, \\
& \leqslant C\|u\|_{H^{1}}\|v\|_{p^{\prime}(\sigma+1)}^{\sigma+1}, \\
& \leqslant C \sqrt{\frac{1}{\min \left\{1, \alpha^{2}\right\}}}\|u\|_{H^{1}(\alpha)}\|v\|_{p^{\prime}(\sigma+1)}^{\sigma+1},
\end{aligned}
$$

i.e.

$$
\left|\int_{\mathbb{R}^{N}} u v^{\sigma+1} \mathrm{~d} x\right| \leqslant C_{\alpha}\|v\|_{p^{\prime}(\sigma+1)}^{2(\sigma+1)},
$$

where $C_{\alpha}=C \max \left\{1, \alpha^{2}\right\} / \min \left\{1, \alpha^{2}\right\} \sim \frac{1}{\alpha^{2}}$ for $\alpha \ll 1$.

By (8), taking $q=p^{\prime}(\sigma+1)$, we obtain

$$
\begin{aligned}
\left|\int_{\mathbb{R}^{N}} u v^{\sigma+1} \mathrm{~d} x\right| & \leqslant C_{\alpha}\|v\|_{H^{1}}^{\frac{p^{\prime}(\sigma+1)-2}{2 p^{\prime}(\sigma+1)} \cdot 2 \cdot 2(\sigma+1)}\|v\|^{\left(1-\frac{p^{\prime}(\sigma+1)-2}{2 p^{\prime}(\sigma+1)} \cdot 2\right) 2(\sigma+1)}, \\
& =C_{\alpha}\|v\|^{\left(1-\frac{p^{\prime}(\sigma+1)-2}{2 p^{\prime}(\sigma+1)} \cdot 2\right) 2(\sigma+1)}\|v\|_{H^{1}}^{2\left(\frac{p^{\prime}(\sigma+1)-2}{p^{\prime}}\right)} .
\end{aligned}
$$

Then (45) yields

$$
\|v\|_{H^{1}}^{2}=\|\nabla v\|^{2}+\|v\|^{2} \leqslant \mathcal{H}_{0}+\mathcal{N}_{0}+C_{\alpha}\left\|v_{0}\right\|^{\left(1-\frac{p^{\prime}(\sigma+1)-2}{2 p^{\prime}(\sigma+1)} \cdot 2\right) 2(\sigma+1)}\|v\|_{H^{1}}^{2\left(\frac{p^{\prime}(\sigma+1)-2}{p^{\prime}}\right)} .
$$

Requiring $2\left(\frac{p^{\prime}(\sigma+1)-2}{p^{\prime}}\right)<2$, we get $\sigma<\frac{2}{p^{\prime}}$. Since $1<p^{\prime} \leqslant 2$, for any $\sigma<2$, we can always find $p, p^{\prime}$ such that the relation $\sigma<\frac{2}{p^{\prime}}$ holds, which implies that the $H^{1}$-norm of the solution is bounded uniformly independent of $t$ by Young's inequality, so we have global existence in the case of $N=2$ and $\sigma<2=\frac{4}{N}$.

Case 3. $N \geqslant 3$ : taking $p, p^{\prime}>1$ in (46), by (10) and (18), we obtain

$$
\begin{aligned}
\|u\|_{p} & \leqslant C\|u\|_{W^{2, m}}, \\
& =C\left\|B\left(|v|^{\sigma+1}\right)\right\|_{W^{2, m}}, \\
& \leqslant C_{\alpha}\left\||v|^{\sigma+1}\right\|_{m}, \\
& =C_{\alpha}\|v\|_{(\sigma+1) m}^{\sigma+1},
\end{aligned}
$$

where

$$
\frac{1}{p}=\frac{1}{m}-\frac{2}{N}>0 \Rightarrow m<\frac{N}{2} .
$$


Plugging into (46) and requiring $m=p^{\prime}$, i.e. $m=\frac{2 N}{N+2}$, we get

$$
\left.\left|\int_{\mathbb{R}^{N}} u\right| v\right|^{\sigma+1} \mathrm{~d} x \mid \leqslant C_{\alpha}\|v\|_{m(\sigma+1)}^{2(\sigma+1)} .
$$

By (8), taking $q=m(\sigma+1)$, we obtain

$$
\|v\|_{m(\sigma+1)} \leqslant C\|v\|^{1-\frac{m(\sigma+1)-2}{2 m(\sigma+1)} N}\|v\|_{H^{1}}^{\frac{m(\sigma+1)-2}{2 m(\sigma+1)} N}
$$

with

$$
0<\frac{m(\sigma+1)-2}{2 m(\sigma+1)} N<1 \Rightarrow \sigma<\frac{4}{N-2} .
$$

Then (45) yields

$$
\|v\|_{H^{1}}^{2}=\|\nabla v(t)\|^{2}+\|v\|^{2} \leqslant \mathcal{H}_{0}+\mathcal{N}_{0}+C_{\alpha}\left\|v_{0}\right\|^{2(\sigma+1)-(\sigma N-2)}\|v\|_{H^{1}}^{\sigma N-2} .
$$

For (64), $\|v\|_{H^{1}}$ is bounded when $\sigma N-2<2$, i.e. $\sigma<\frac{4}{N}$. Therefore, the $H^{1}$ norm of the solution $v$ is bounded uniformly independent of time $t$, so we can conclude that we have global solution for any $1 \leqslant \sigma<\frac{4}{N}$.

\section{Modulation theory}

As one can see from the proof of global existence of system (4), the uniform bound of $H^{1}$-norm of the solution depends on a constant $C_{\alpha} \sim \frac{1}{\alpha^{2}}$ when $\alpha$ is very small, in other words, in the limit $\alpha \rightarrow 0^{+}$, this upper bound will grow to infinity. One expects this result since as $\alpha$ goes to zero, we will recover the classical NLS and we will 'lose' these improvements on the range of the exponent of $\sigma$. In fact, when the parameter $\alpha$ goes to zero, one can regard system (4) as a perturbation of the classical NLS, since we can rewrite system (4) as

$$
\mathrm{i} v_{t}+\Delta v+|v|^{2 \sigma} v+\alpha^{2} \Delta u v=0 .
$$

where $u=\left(I-\alpha^{2} \triangle\right)^{-1}\left(|v|^{\sigma+1}\right)$.

There have been many works on perturbed NLS in the critical case $\sigma=\frac{2}{N}$ (see, e.g., $[13,16,17]$, and references therein). In this section, we will apply modulation theory (see, e.g., $[20,33,35]$ for references about modulation theory) to the classical critical case $\sigma=1, N=2$. In this case the classical NLS blows up for certain initial data, and modulation theory tries to explain the role of the regularization in preventing the formation of a singularity near the critical values of the initial data which blow up in the classical case. The intuition of modulation theory is that the energy near a singularity is equal to the power of the Townes soliton and the profiles of the solutions are asymptotic to some rescaled profiles of the Townes soliton. With modulation theory, one can reduce the perturbed system (65) into a simpler system of ordinary differential equations that do not depend on the spatial variables, and they are supposed to be easier to analyse both analytically and numerically.

First we review some main results on modulation theory for the unperturbed critical NLS following [20]. As stated in [20], most of the results presented in this section have not been made rigorous at present.

In the case of self-focusing the amount of power which goes into the singularity is equal to the critical power $N_{\mathrm{c}}=\|R\|_{2}^{2}$, where $R$ is the Townes soliton (2), the beam separates into two components as it propagates,

$$
v=v_{s}+v_{\text {back }}
$$

where $v_{\mathrm{s}}$ is the high intensity inner core of the beam which self-focuses towards its centre axis and $v_{\text {back }}$ is the low intensity outer part which propagates forward following the usual linear 
propagation mode. Close enough to the singularity, $v_{\mathrm{s}}$ has only small excess power above the critical one and approaches the radially symmetric asymptotic profile:

$$
v_{s}(x, t)=\frac{1}{L(t)} V(\tau, \xi) \exp \left(\mathrm{i}\left(\tau+\frac{L_{t}}{L} \frac{r^{2}}{4}\right)\right), \quad \arg V(\tau, 0)=0,
$$

where $L(t)$ is a yet to be determined function that is used to rescale and the independent variables, $\xi=\left(\xi_{1}, \xi_{2}\right), x=\left(x_{1}, x_{2}\right)$ with

$$
\xi=\frac{x}{L}, \quad \frac{\mathrm{d} \tau}{\mathrm{d} t}=\frac{1}{L^{2}} .
$$

Then the reduced system for unperturbed critical NLS is

$$
L_{t t}=-\frac{\beta}{L^{3}}, \quad \beta_{t}=-\frac{\mathrm{e}^{-\frac{\pi}{\sqrt{\beta}}}}{L^{2}},
$$

where $L(t)$ is the scaling factor and $\beta$ is proportional to the excess of the power near a singularity: $\beta=M\left(N-N_{c}\right)$ for constant $M=\frac{1}{4} \int_{0}^{\infty} R(\rho)^{2} \rho^{3} \mathrm{~d} \rho \approx 0.55$.

For a general perturbed critical NLS of the form

$$
\mathrm{i} v_{t}+\Delta v+|v|^{2} v+\epsilon F\left(v, v_{t}, \nabla v, \cdots\right)=0, \quad|\epsilon| \ll 1,
$$

where $F$ is an even function in $x$, the modulation theory is valid when the following three conditions hold. Observe that in our system (65) $\epsilon$ is the parameter $\alpha^{2}$.

Condition 1. The focusing part of the solution is close to the asymptotic profile

$$
v_{s}(t, x) \sim \frac{1}{L(t)} V(\tau, \xi) \exp \left[\mathrm{i} \tau(t)+\mathrm{i} \frac{L_{t}}{L} \frac{r^{2}}{4}\right],
$$

where

$$
\xi=\frac{x}{L}, \quad r^{2}=x_{1}^{2}+x_{2}^{2}, \quad \frac{\mathrm{d} \tau}{\mathrm{d} t}=\frac{1}{L(t)^{2}}
$$

and $V=R+O(\beta, \epsilon), \beta=-L^{3} L_{t t}$ and $R$ is the Townes soliton given in (2).

Condition 2. The power is close to critical

$$
\left.\left|\frac{1}{2 \pi} \int\right| v_{s}\left(t, x_{1}, x_{2}\right)\right|^{2} \mathrm{~d} x_{1} \mathrm{~d} x_{2}-N_{\mathrm{c}} \mid \ll 1,
$$

or, equivalently,

$$
|\beta(t)| \ll 1,
$$

where $N_{\mathrm{c}}=\frac{1}{2 \pi} \int_{\mathbb{R}^{2}} R\left(x_{1}, x_{2}\right)^{2} \mathrm{~d} x_{1} \mathrm{~d} x_{2}=\|R\|_{2}^{2}$ is the threshold energy of blow-up.

Condition 3. The perturbation $\epsilon F$ is small in comparison with the other terms, i.e.

$$
|\epsilon F| \ll|\Delta v|, \quad|\epsilon F| \ll|v|^{3} .
$$

The following proposition is given in [20].

Proposition 11. If conditions 1-3 hold, self-focusing in the perturbed critical NLS (69) is given to leading order by the reduced system

$$
\beta_{t}+\frac{\mathrm{e}^{-\frac{\pi}{\sqrt{\beta}}}}{L^{2}}=\frac{\epsilon}{2 M}\left(f_{1}\right)_{t}-\frac{2 \epsilon}{M} f_{2}, \quad L_{t t}=-\frac{\beta}{L^{3}} .
$$


The auxiliary functions $f_{1}, f_{2}$ are given by

$f_{1}(t)=2 L(t) \operatorname{Re}\left[\frac{1}{2 \pi} \int_{\mathbb{R}^{2}} F\left(\psi_{R}\right) \exp (-\mathrm{i} S)[R(\rho)+\rho \nabla R(\rho)] \mathrm{d} x_{1} \mathrm{~d} x_{2}\right]$,

$f_{2}(t)=\operatorname{Im}\left[\frac{1}{2 \pi} \int_{\mathbb{R}^{2}} \psi_{R}^{*} F\left(\psi_{R}\right) \mathrm{d} x_{1} \mathrm{~d} x_{2}\right]$

where $\psi_{R}=\frac{1}{L} R(\rho) \exp (\mathrm{i} S), R$ is the Townes soliton given in (2), $\rho=\frac{r}{L}, S=\tau(t)+\frac{L_{t}}{L} \frac{r^{2}}{4}$, $\frac{\mathrm{d} \tau}{\mathrm{d} t}=\frac{1}{L^{2}}, M=\frac{1}{4} \int_{0}^{\infty} R(\rho)^{2} \rho^{3} \mathrm{~d} \rho \approx 0.55$.

Furthermore, if $F$ is a conservative perturbation, i.e.

$$
\operatorname{Im} \int_{\mathbb{R}^{2}} v^{*} F(v) \mathrm{d} x_{1} \mathrm{~d} x_{2}=0
$$

then $f_{2}=0$.

In general, at the onset of self-focusing only condition 3 holds. Therefore, if the power is above critical $N_{\mathrm{c}}$ the solution will initially self-focus as in the unperturbed critical NLS. As a result, near the time of blow-up in the absence of the perturbation, conditions 1 and 2 will also be satisfied.

As studied in [20], various conservative perturbations of critical NLS, for instance, selffocusing in fibre arrays (see [2-5,32,49] and references therein) and small dispersive fifthpower nonlinear perturbation to the classical NLS [35], have a generic form

$$
f_{1} \sim-\frac{C}{L^{2}}, \quad C=\text { constant }
$$

which results in a canonical focusing-defocusing oscillation.

Now, let us calculate the reduced equations of the SH system.

Apply the above results to system (4) or (65). Assuming first that system (65) satisfies all three conditions above we will carry out the calculations below. Numerical computations will be presented in a further paper. Observe that our perturbation term is a nonlocal operator

$$
F(v)=(\triangle u) v,
$$

where $u$ satisfies the equation $u-\alpha^{2} \triangle u=|v|^{2}$.

Since

$$
\psi_{R}(x)=\frac{1}{L} R\left(\frac{x}{L}\right) \exp (\mathrm{i} S)
$$

we have

$$
F\left(\psi_{R}\right)(x)=\left(\triangle u_{R}\right) \psi_{R}(x),
$$

where $u_{R}$ satisfies

$u_{R}(x)-\alpha^{2} \triangle u_{R}(x)=\left|\psi_{R}(x)\right|^{2}=\left|\frac{1}{L} R\left(\frac{x}{L}\right) \exp (\mathrm{i} S)\right|^{2}=\frac{1}{L^{2}}\left|R\left(\frac{x}{L}\right)\right|^{2}$.

Written in a more general form

$$
\left(I-\alpha^{2} \triangle\right) u(x)=g\left(\frac{x}{L}\right), \quad x \in \mathbb{R}^{N}
$$

then $u(x)=\left(B_{\frac{\alpha}{L}} * g\right)\left(\frac{x}{L}\right)$, where $B_{\frac{\alpha}{L}}$ is the modified Bessel potential:

$$
B_{\frac{\alpha}{L}}(x)=\left(\frac{1}{2 \alpha^{2}}\right)^{\frac{N}{2}} \int_{0}^{\infty} \frac{\mathrm{e}^{-s} \mathrm{e}^{-\frac{|x|^{2}}{4 s(\alpha / L)^{2}}}}{s^{N / 2}} \mathrm{~d} s .
$$


Returning to equation (82), now we have $g(\cdot)=\frac{1}{L^{2}} R(\cdot)^{2}$, so we can write the solution $u_{R}(x)$ as

$$
\begin{aligned}
u_{R}(x) & =\left(B_{\frac{\alpha}{L}} * \frac{1}{L^{2}} R^{2}\right)\left(\frac{x}{L}\right)=\frac{1}{L^{2}}\left(B_{\frac{\alpha}{L}} * R^{2}\right)\left(\frac{x}{L}\right) \\
& =\frac{1}{L^{2}} \frac{1}{2 \alpha^{2}} \int_{\mathbb{R}^{2}}\left(\int_{0}^{\infty} \frac{\mathrm{e}^{-s} \mathrm{e}^{-\frac{|x / L-y|^{2}}{4 s(\alpha / L)^{2}}}}{s} \mathrm{~d} s\right) R^{2}(y) \mathrm{d} y_{1} \mathrm{~d} y_{2},
\end{aligned}
$$

where $y=\left(y_{1}, y_{2}\right)$.

Plugging the above into (81) and using (82), we get

$$
\begin{aligned}
F\left(\psi_{R}\right)(x) & =\left(\triangle u_{R}(x)\right) \psi_{R}(x)=\frac{1}{\alpha^{2}}\left(u_{R}(x)-\left|\psi_{R}(x)\right|^{2}\right) \psi_{R}(x), \\
& =\frac{1}{\alpha^{2}}\left(u_{R}(x)-\frac{1}{L^{2}} R^{2}\left(\frac{x}{L}\right)\right)\left(\frac{1}{L} R\left(\frac{x}{L}\right) \mathrm{e}^{\mathrm{i} S}\right) .
\end{aligned}
$$

Now we can calculate the term $f_{1}$

$$
\begin{aligned}
f_{1} & =\frac{L}{\pi} \operatorname{Re} \int_{\mathbb{R}^{2}} F\left(\psi_{R}(x)\right) \exp (-\mathrm{i} S)\left(R(\rho)+\rho R_{\rho}\right) \mathrm{d} x_{1} \mathrm{~d} x_{2}, \\
& =\frac{L}{\pi} \int_{\mathbb{R}^{2}} \frac{1}{\alpha^{2}}\left(u_{R}(x)-\frac{1}{L^{2}} R^{2}(\rho)\right)\left(\frac{1}{L} R(\rho)\right)\left(R(\rho)+\rho R_{\rho}\right) \mathrm{d} x_{1} \mathrm{~d} x_{2}, \\
& =\frac{1}{\pi \alpha^{2}} \int_{\mathbb{R}^{2}} u_{R}(x) R(\rho)\left(R(\rho)+\rho R_{\rho}\right) \mathrm{d} x_{1} \mathrm{~d} x_{2}-\frac{1}{\pi \alpha^{2}} \frac{1}{L^{2}} \int_{\mathbb{R}^{2}} R^{3}(\rho)\left(R(\rho)+\rho R_{\rho}\right) \mathrm{d} x_{1} \mathrm{~d} x_{2}, \\
& =I_{1}-I_{2} .
\end{aligned}
$$

It is easy to see that the second integral $I_{2}$ is a constant that does not depend on $L$. Indeed, applying the change of variables: $\xi_{1}=\frac{x_{1}}{L}, \xi_{2}=\frac{x_{2}}{L}$, since $\rho=\frac{r}{L}=\frac{\sqrt{x_{1}^{2}+x_{2}^{2}}}{L}=\sqrt{\xi_{1}^{2}+\xi_{2}^{2}}$, we get

$$
I_{2}=\frac{1}{\pi \alpha^{2}} \int_{\mathbb{R}^{2}} R^{3}(\rho)\left(R(\rho)+\rho R_{\rho}(\rho)\right) \mathrm{d} \xi_{1} \mathrm{~d} \xi_{2}=c_{0},
$$

where $c_{0}$ is a constant that does not depend on $L$.

Next, let us look at the first integral $I_{1}$, plugging the result of (86) into $I_{1}$, we get

$$
\begin{aligned}
I_{1}= & \frac{1}{\pi \alpha^{2}} \int_{\mathbb{R}^{2}} u_{R}(x) R(\rho)\left(R(\rho)+\rho R_{\rho}\right) \mathrm{d} x_{1} \mathrm{~d} x_{2}, \\
= & \frac{1}{\pi \alpha^{2}} \int_{\mathbb{R}^{2}} \frac{1}{L^{2}} \frac{1}{2 \alpha^{2}} \int_{\mathbb{R}^{2}}\left(\int_{0}^{\infty} \frac{\mathrm{e}^{-s} \mathrm{e}^{-\frac{|x / L-y|^{2}}{4 s(\alpha / L)^{2}}}}{s} \mathrm{~d} s\right) R^{2}(y) \mathrm{d} y_{1} \mathrm{~d} y_{2} R(\rho)(R(\rho) \\
& \left.+\rho R_{\rho}\right) \mathrm{d} x_{1} \mathrm{~d} x_{2}, \\
= & \frac{1}{2 \pi} \frac{1}{\alpha^{4}} \frac{1}{L^{2}} \int_{\mathbb{R}^{2}} \int_{\mathbb{R}^{2}} \int_{0}^{\infty} \frac{\mathrm{e}^{-s} \mathrm{e}^{-\frac{|x / L-y|^{2}}{4 s(\alpha / L)^{2}}}}{s} \mathrm{~d} s R^{2}(y) \mathrm{d} y_{1} \mathrm{~d} y_{2} R(\rho)\left(R(\rho)+\rho R_{\rho}\right) \mathrm{d} x_{1} \mathrm{~d} x_{2} .
\end{aligned}
$$

Applying the change of variables: $\xi=\left(\xi_{1}, \xi_{2}\right)=\left(\frac{x_{1}}{L}, \frac{x_{2}}{L}\right)$, we will then have the following integral:

$I_{1}=\frac{1}{2 \pi} \frac{1}{\alpha^{4}} \int_{\mathbb{R}^{2}} \int_{\mathbb{R}^{2}} \int_{0}^{\infty} \frac{\mathrm{e}^{-s} \mathrm{e}^{-\frac{|\xi-y|^{2}}{4 s(\alpha / L)^{2}}}}{s} \mathrm{~d} s R^{2}(y) \mathrm{d} y_{1} \mathrm{~d} y_{2} R(\rho)\left(R(\rho)+\rho R_{\rho}\right) \mathrm{d} \xi_{1} \mathrm{~d} \xi_{2}$ 
Therefore, $f_{1}$ can be written as

$f_{1}=\frac{1}{2 \pi} \frac{1}{\alpha^{4}} \int_{\mathbb{R}^{2}} \int_{\mathbb{R}^{2}} \int_{0}^{\infty} \frac{\mathrm{e}^{-s} \mathrm{e}^{-\frac{|\xi-y|^{2}}{4 s(\alpha / L)^{2}}}}{s} \mathrm{~d} s R^{2}(y) \mathrm{d} y_{1} \mathrm{~d} y_{2} R(\rho)\left(R(\rho)+\rho R_{\rho}\right) \mathrm{d} \xi_{1} \mathrm{~d} \xi_{2}+c_{0}$.

Hence we have the reduced system

$$
\begin{aligned}
& \beta_{t}+\frac{\mathrm{e}^{-\frac{\pi}{\sqrt{\beta}}}}{L^{2}}=\frac{\alpha^{2}}{2 M}\left(f_{1}\right)_{t}, \\
& L_{t t}=-\frac{\beta}{L^{3}},
\end{aligned}
$$

where $f_{1}$ is given in the form of (93). It is worth stressing that the right-hand side of equation (94) is different from that of (68), which indicates the rate of regularization parameter $\alpha$.

Now one needs to study the ODE (94) with $f_{1}$ given in (93). The explicit form of the function $f_{1}$ is much more complicated when compared with the generic form of (78). The idea is to show that for small $L$ this additional term on the right-hand side of the first equation of (94) will prevent the singularity formation, i.e. prevent $L$ from tending to zero as time evolves. However, we do not provide here a detailed analysis of systems (93) and (94), since it is out of the scope of this paper. More detailed study on modulation theory and reduced system will be reported in a forthcoming paper.

\section{Acknowledgments}

The authors would like to thank the anonymous referees for their valuable comments and suggestions. This work was supported in part by the NSF grant nos DMS-0504619 and DMS0708832, the BSF grant no 2004271 and the ISF grant no $120 / 06$.

\section{References}

[1] Ablowitz M J and Segur H 1981 Solitons and The Inverse Scattering Transform (SIAM Studies in Applied Mathematics vol 4) (Philadelphia, PA: SIAM)

[2] Aceves A, De Angelis C, Luther G, Rubenchik A and Turitsyn S 1995 All-optical-switching and pulse amplification and steering in nonlinear fiber arrays Phys. Rev. Lett. 75 73-6

[3] Aceves A, De Angelis C, Rubechik A and Turitsyn S 1995 Multidimensional solitons in fiber array Opt. Lett. 19 329-31

[4] Aceves A, De Angelis C, Luther G, Rubenchik A and Turitsyn S 1995 Energy localization in nonlinear fiber arrays: collapse effect compressor Physica D 87 262-72

[5] Aceves A, De Angelis C, Luther G, Rubenchik A and Turitsyn S 1995 Optical pulse compression using fiber arrays Opt. Fiber Technol. $1244-6$

[6] Adams R A 1975 Sobolev Spaces (New York: Academic)

[7] Brezis H and Gallouet T 1980 Nonlinear Schrödinger evolution equations Nonlinear Anal. Theory Methods Appl. 4 677-81

[8] Cao Y, Lunasin E M and Titi E S 2006 Global well-posedness of three-dimensional viscous and inviscid simplified Bardina turbulence models Commun. Math. Sci. 4 823-48

[9] Castella F $1997 L^{2}$-solutions to the Schödinger-Poisson system: existence, uniqueness, time behavior, and smoothing effects Math. Models Methods Appl. Sci. 7 1051-83

[10] Cazenave T 2003 Semilinear Schrödinger Equations (Courant Lecture Notes in Mathematics) (Providence, RI: American Mathametical Society)

[11] Chen S, Foias C, Holm D D, Olson E, Titi E S and Wynne S 1999 The Camassa-Holm equations and turbulence Physica D 133 49-65

[12] Cheskidov A, Holm D D, Olson E and Titi E S 2005 On a Leray- $\alpha$ model of turbulence Proc. R. Soc. Lond. A $461629-49$ 
[13] Fibich G 2001 Self-focusing in the damped nonlinear Schrödinger equation SIAM J. Appl. Math. 61 1680-705

[14] Fibich G 1996 Adiabatic law for self-focusing of optical beams Opt. Lett. 21 1735-7

[15] Fibich G 1996 Small beam nonparaxiality arrests self-focusing of optical beams Phys. Rev. Lett. 76 4356-9

[16] Fibich G, Ilan B and Papanicolaou G 2002 Self-focusing with fourth-order dispersion SIAM J. Appl. Math. 62 1437-62

[17] Fibich G and Levy D 1998 Self-focusing in the complex Ginzburg-Landau limit of the critical nonlinear Schrödinger equation Phys. Lett. A 249 286-94

[18] Fibich G and Merle F 2001 Self-focusing on bounded domains Physica D 155 132-58

[19] Fibich G and Papanicolaou G 1998 A modulation method for self-focusing in the perturbed critical nonlinear Schrödinger equation Phys. Lett. A 239 167-73

[20] Fibich G and Papanicolaou G 1999 Self-focusing in the perturbed and unperturbed nonlinear Schrödinger equation in critical dimenstion SIAM J. Appl. Math. $60183-240$

[21] Foias C, Holm D D and Titi E S 2002 The three dimensional viscous Camassa-Holm equations and their relation to the Navier-Stokes equations and turbulence theory J. Dyn. Diff. Eqns 14 1-35

[22] Galdi G 1994 An Introduction to the Mathematical Theory of the Navier-Stokes Equations vol 1 (Berlin: Springer)

[23] Ginibre J and Velo G 1979 On a class of nonlinear Schrödinger equations: I. The Cauchy problem, general case J. Funct. Anal. 32 1-32

[24] Glassey R T 1977 On the blowing-up of solutions to the Cauchy problem for the nonlinear Schrödinger equation J. Math. Phys. 18 1794-7

[25] Grafakos L 2003 Classical and Modern Fourier Analysis (Englewood Cliffs, NJ: Prentice Hall)

[26] Hayashi N and Ozawa T 1989 Smoothing effect for some Schrödinger equations J. Funct. Anal. $85307-48$

[27] Holm D D, Marsden J and Ratiu T 2002 The Euler-Poincaré equations in geophysical fluid dynamics LargeScale Atmosphere-Ocean Dynamics: H. Geometric Methods and Models (Cambridge: Cambridge University Press) pp 251-300

[28] Ilyin A, Lunasin E M and Titi E S 2006 A modified-Leray- $\alpha$ subgrid scale model of turbulence Nonlinearity 19 879-97

[29] Kato T 1987 On nonlinear Schrödinger equations Ann. Inst. H Poincaré Phys. Theor. 46 113-29

[30] Kelley P L 1965 Self-focusing of optical beams Phys. Rev. Lett. 15 1005-8

[31] Kupferman R, Mangoubi C and Titi E S 2008 A Beale-Kato-Majda breakdown criterion for an Oldroyd-B fluid in the creeping flow regime Commun. Math. Sci. 6 235-56

[32] Laedke E, Spatschek H and Turitsyn S 1991 Analytics criterion for soliton instability in a nonlinear fiber array Phys. Rev. E 52 5549-54

[33] Landman M, Papanicolaou G, Sulem C and Sulem P 1988 Rate of blowup for solutions of the nonlinear Schrödinger equation at critical dimension Phys. Rev. A $383837-43$

[34] Lemesurier B, Papanicolaou G, Sulem C and Sulem P 1988 Local structure of the self-focusing singularity of the nonlinear Schrödinger equation Physica D 32 210-26

[35] Malkin V 1993 On the analytical theory for stationary self-focusing of radiation Physica D 64 251-66

[36] Merle F 1993 Determination of blow-up solutions with minimal mass for nonlinear Schrödinger equation with critical power Duke Math. J. 69 427-54

[37] Merle F and Raphael P 2005 On a sharp lower bound on the blow-up rate for the $\iota^{2}$ critical nonlinear Schrödinger equation J. Am. Math. Soc. $1937-90$

[38] Merle F and Tsutsumi Y $1990 L^{2}$ concentration of blow-up solutions for the nonlinear Schrödinger equation with critical power nonlinearity J. Diff. Eqns 84 205-14

[39] Mitrović D and Žubrinić D 1997 Fundamentals of Applied Functional Analysis (Pitman Monographs and Surveys in Pure and Applied Mathematics vol 91) (London: Longmans Green)

[40] Moroz I M, Penrose R and Tod P 1998 Spherically-symmetric solutions of the Schrödinger-Newton equation Class. Quantum Grav. 15 2733-42

[41] Penrose R 1996 On gravity's role in quantum state reduction Gen. Rel. Grav. 28 581-600

[42] Robertshaw O and Tod P 2006 Lie point symmetries and an approximate solution for the Schrödinger-Newton equations Nonlinearity $191507-14$

[43] Ruffini R and Bonazzola S 1969 Systems of self-gravitating particles in general relativity and the concept of an equation of state Phys. Rev. 187 1767-83

[44] Stein E 1970 Singular Integrals and Differentiability Properties of Functions (Princeton, NJ: Princeton University Press)

[45] Sulem C and Sulem P L 1999 The Nonlinear Schödinger Equation Self-Focusing and Wave Collapse (Applied Mathematical Sciences vol 139) (Berlin: Springer)

[46] Taniuti T and Washimi H 1968 Self-trapping and instability of hydromagnetic waves along the magnetic field in a cold plasma Phys. Rev. Lett. 21 209-12 
[47] Tod P and Moroz I M 1999 An analytical approach to the Schrödinger-Newton equations Nonlinearity 12 201-16

[48] Weinstein M I 1983 Nonlinear Schrödinger equations and sharp interpolation estimates Commun. Math. Phys. $87567-76$

[49] Weinstein M and Yeary B 1996 Excitation and dynamics of pulses in coupled fiber arrays Phys. Lett. A 222 157-62

[50] Yudovich V I 1963 Non-stationary flow of an ideal incompressible liquid Zh. Vychils. Mat. 3 1032-66

Yudovich V I 1963 Comput. Math. Math. Phys. 3 1407-56 (Eng. Transl.)

[51] Yudovich V I 1989 The Linearization Method in Hydrodynamical Stability Theory (Translations of Mathematical Monographs vol 74) (Providence, RI: American Mathematical Society)

[52] Zakharov V E, Sobolev V V and Synakh V C 1971 Behavior of light beams in nonlinear media Sov. Phys._JETP. $3377-81$ 